\title{
Overall Prevalence and Prevalence Compared among Psoriasis Treatments of Onychomycosis in Patients with Nail Psoriasis and Fungal Involvement
}

\section{Leena Chularojanamontri (iD), Penvadee Pattanaprichakul $(\mathbb{D}$, Charussri Leeyaphan (iD), Panittra Suphatsathienkul $(\mathbb{D}$, Supisara Wongdama $\mathbb{D}$, and Sumanas Bunyaratavej}

Department of Dermatology, Faculty of Medicine Siriraj Hospital, Mahidol University, Bangkok, Thailand

Correspondence should be addressed to Sumanas Bunyaratavej; consultskin@yahoo.com

Received 3 June 2021; Revised 28 November 2021; Accepted 30 November 2021; Published 13 December 2021

Academic Editor: Francesca Mancianti

Copyright (c) 2021 Leena Chularojanamontri et al. This is an open access article distributed under the Creative Commons Attribution License, which permits unrestricted use, distribution, and reproduction in any medium, provided the original work is properly cited.

\begin{abstract}
Background. Whether nail psoriasis can increase the risk of onychomycosis is still being debated, and data relating to the prevalence of onychomycosis among psoriasis patients receiving different treatments is limited. Objectives. To investigate the overall prevalence and prevalence compared among psoriasis treatments of onychomycosis in patients with nail psoriasis and fungal involvement. Methods. A prospective study of three groups of nail psoriasis being treated with only topical medication, methotrexate, or biologics (25 patients per group, 150 nails) was conducted at Siriraj Hospital (Bangkok, Thailand) during November 2018 to September 2020. Demographic data, psoriasis severity, and nail psoriasis severity were recorded. The nail most severely affected with psoriasis on each hand was selected for mycological testing. Potassium hydroxide, periodic acid-Schiff stain, and fungal culture were performed. Results. The prevalence of onychomycosis in nail psoriasis was 35.3\%. Among the treatment groups, the prevalence of onychomycosis was significantly higher in the methotrexate group than in the topical treatment and biologic treatment groups $(p=0.014)$. Candida spp. was the main causative organism, followed by Trichophyton rubrum. Thumb was most commonly affected (59.3\%). The most common abnormality of the nail matrix and the nail bed was pitted nail (71.3\%) and onycholysis (91.3\%), respectively. Multivariate analysis revealed diabetes, wet-work exposure, and methotrexate treatment to be predictors of onychomycosis. Conclusions. Several factors, including psoriasis treatment, were shown to increase the risk of onychomycosis in nail psoriasis. Further research is needed to determine whether biologic agents, especially interleukin-17 inhibitors, can increase risk of onychomycosis and Candida infection/colonization of the nails.
\end{abstract}

\section{Introduction}

Psoriasis is a multifactorial chronic disorder that has an etiopathogenesis that derives from the alteration of signaling pathways, which leads to a defect in the functional and structural properties of the skin [1]. It can cause nail pathology on both the hands and feet. The reported prevalence of nail involvement in patients with psoriasis varied considerably from $15 \%$ to $86 \%[2,3]$. The presence of nail involvement was reported to be a predictor of psoriatic arthritis, and it was also found that nail involvement may occur a few years before the development of joint disease [4]. Psoriasis can affect the nail matrix and nail bed resulting in several clinical presentations, including pitting, crumbling, onycholysis, and subungual hyperkeratosis [5].

Onychomycosis is a fungal nail infection that is caused by dermatophytes, yeasts, and filamentous fungi. It is a common nail disease that accounts for $50 \%$ of nail disorders. Its prevalence in the general population and in patients with psoriasis can reach up to $30 \%$ and $56 \%$, respectively $[5,6]$. Clinical features of nail psoriasis and onychomycosis may overlap, and pathologies of both diseases may occur in the same patient. Several factors, including nail pathologies, patient behavior, immune status, and treatments for psoriasis, may contribute to the development of onychomycosis in nail psoriasis. 
Topical treatments, such as steroids, vitamin D3 analogs, tazarotene, trifarotene, topical calcineurin inhibitors, and 5fluorouracil, can be used to treat nail psoriasis [7-9]. For patients with severe skin involvement and nail psoriasis, conventional systemic treatments (ciclosporin, methotrexate, and acitretin), small molecule drugs, and biologics are recommended. Due to their immunosuppressive properties, ciclosporin, methotrexate, and biologics may aggravate onychomycosis in nail psoriasis [3]. On the other hand, there is evidence that vitamin $\mathrm{A}$ and its active metabolite, all-transretinoic acid, exert host-protective effects in infections and direct fungistatic effect against Candida albicans [9-11]. Recently, small molecule drugs, such as apremilast and tofacitinib, have been shown to be effective for treating nail psoriasis $[3,12]$.

Although several studies have investigated the prevalence of onychomycosis in patients with nail psoriasis, few studies have addressed its prevalence among patients receiving different treatments for psoriasis. Among those studies, a cross-sectional study reported a prevalence of onychomycosis of $34.8 \%(8 / 23)$ among patients not being treated with immunosuppressive agents, and yeasts, and filamentous fungi were the predominant pathogens [13]. Other studies reported that factors affecting the immune status, including diabetes, administration of topical corticosteroids, and systemic treatments for psoriasis, were risk factors for onychomycosis in patients with psoriasis $[6,14]$. A randomized prospective open-label study reported that the risk of onychomycosis in psoriasis patients receiving treatment with an antitumor necrosis factor (anti-TNF) was $20.3 \%$ compared to $13.9 \%$ in patients that did not receive any biological agents [15]. Moreover and importantly, published data relating to the prevalence of onychomycosis among patients receiving anti-interleukin- (IL-) 17, in which mucocutaneous candidiasis is a side effect of concern, is comparatively scarce [16].

Thus, the aim of this study was to investigate the overall prevalence and prevalence of onychomycosis among different treatments for psoriasis, including topical medication, methotrexate, or biologic therapy in patients with nail psoriasis. Our secondary objective was to identify significant risk factors for developing onychomycosis in this patient population.

\section{Materials and Methods}

This prospective study was conducted at the Department of Dermatology, Faculty of Medicine Siriraj Hospital, Mahidol University, Bangkok, Thailand during November 2018 to September 2020. The inclusion criteria were psoriasis patients with fingernail pathologies aged 18 years or older who attended the outpatient dermatology clinic at our center and who were being treated with only topical treatment, methotrexate, or biologic agents for at least four weeks. The exclusion criteria were (i) patients with a history of receiving any topical or systemic antifungal agents during the 12 -week period prior to the start of this study, (ii) patients taking other immunosuppressant drugs, (iii) pregnant or lactating patients, and/or (iv) patients with other nail diseases. All patients who voluntarily agreed to participate in
TABLE 1: Demographic and clinical characteristics of nail psoriasis patients with onychomycosis.

\begin{tabular}{|c|c|}
\hline Characteristics $(N=75)$ & Values \\
\hline \multicolumn{2}{|l|}{ Gender, $n(\%)$} \\
\hline Female & $38(50.7 \%)$ \\
\hline Male & $37(49.3 \%)$ \\
\hline Age (years), mean $\pm S D$ & $46.8 \pm 15.3$ \\
\hline Body mass index $\left(\mathrm{kg} / \mathrm{m}^{2}\right)$, mean $\pm \mathrm{SD}$ & $25.0 \pm 5.0$ \\
\hline Duration of psoriasis (years), median (IQR) & $\begin{array}{l}11.0(7.0 \\
20.0)\end{array}$ \\
\hline \multicolumn{2}{|l|}{ Psoriasis types, $n(\%)$} \\
\hline Plaque & $64(85.3 \%)$ \\
\hline Guttate & $7(9.3 \%)$ \\
\hline Erythrodermic & $3(4.0 \%)$ \\
\hline Pustular & $1(1.3 \%)$ \\
\hline Psoriatic arthritis, $n(\%)$ & $13(17.3 \%)$ \\
\hline \multicolumn{2}{|l|}{ Occupation, $n(\%)$} \\
\hline No increased risk of hand exposure to water ${ }^{\dagger}$ & $40(53.3 \%)$ \\
\hline Increased risk of hand exposure to water ${ }^{\ddagger}$ & $35(46.7 \%)$ \\
\hline Patient right-handed, $n$ (\%) & $68(90.7 \%)$ \\
\hline Patient left-handed, $n(\%)$ & $7(9.3 \%)$ \\
\hline $\begin{array}{l}\text { Handwashing frequency (times/day), } \\
\text { median (IQR) }\end{array}$ & $6.0(4.0,10.0)$ \\
\hline \multicolumn{2}{|l|}{ Current psoriasis severity } \\
\hline $\begin{array}{l}\text { Psoriasis Area and Severity Index score, } \\
\text { median (IQR) }\end{array}$ & $5.0(3.2,10.2)$ \\
\hline Characteristics of fingernail pathologies $(N=150)$ & Values \\
\hline \multicolumn{2}{|l|}{ Nail severity } \\
\hline $\begin{array}{l}\text { Nail Psoriasis Severity Index score }(0-8) \text {, } \\
\text { median (IQR) }\end{array}$ & $4.0(2.0,4.0)$ \\
\hline $\begin{array}{l}\text { Nijmegen-Nail Psoriasis Activity Index } \\
\text { tool }(0-15) \text {, median (IQR) }\end{array}$ & $4.0(3.0,5.0)$ \\
\hline \multicolumn{2}{|l|}{ Most severely affected fingernail, $n(\%)$} \\
\hline Thumb & $89(59.3 \%)$ \\
\hline Index finger & $22(14.7 \%)$ \\
\hline Middle finger & $16(10.7 \%)$ \\
\hline Ring finger & $13(8.7 \%)$ \\
\hline Little finger & $10(6.7 \%)$ \\
\hline \multicolumn{2}{|l|}{ Nail matrix pathology, $n(\%)$} \\
\hline Pitting & $107(71.3 \%)$ \\
\hline Leukonychia & $69(46.0 \%)$ \\
\hline Crumbling & $55(36.7 \%)$ \\
\hline Red spots lunula & $2(1.3 \%)$ \\
\hline \multicolumn{2}{|l|}{ Nail bed pathology, $n(\%)$} \\
\hline Onycholysis & $137(91.3 \%)$ \\
\hline Subungual hyperkeratosis & $53(35.3 \%)$ \\
\hline Oil drop & $30(20.0 \%)$ \\
\hline Splinter hemorrhage & $29(19.3 \%)$ \\
\hline Beau lines, $n(\%)$ & $19(12.7 \%)$ \\
\hline Paronychial involvement, $n(\%)$ & $73(48.7 \%)$ \\
\hline
\end{tabular}

${ }^{\dagger}$ Driver, lawyer, merchant, collegian, teacher, or retired. ${ }^{\ddagger}$ Housekeeper, farmer, fisherman, mechanic, builder, or barber. Abbreviations: SD: standard deviation; IQR: interquartile range. 
TABLE 2: Results of potassium hydroxide $(\mathrm{KOH})$, periodic acid-Schiff (PAS) stain, and culture techniques.

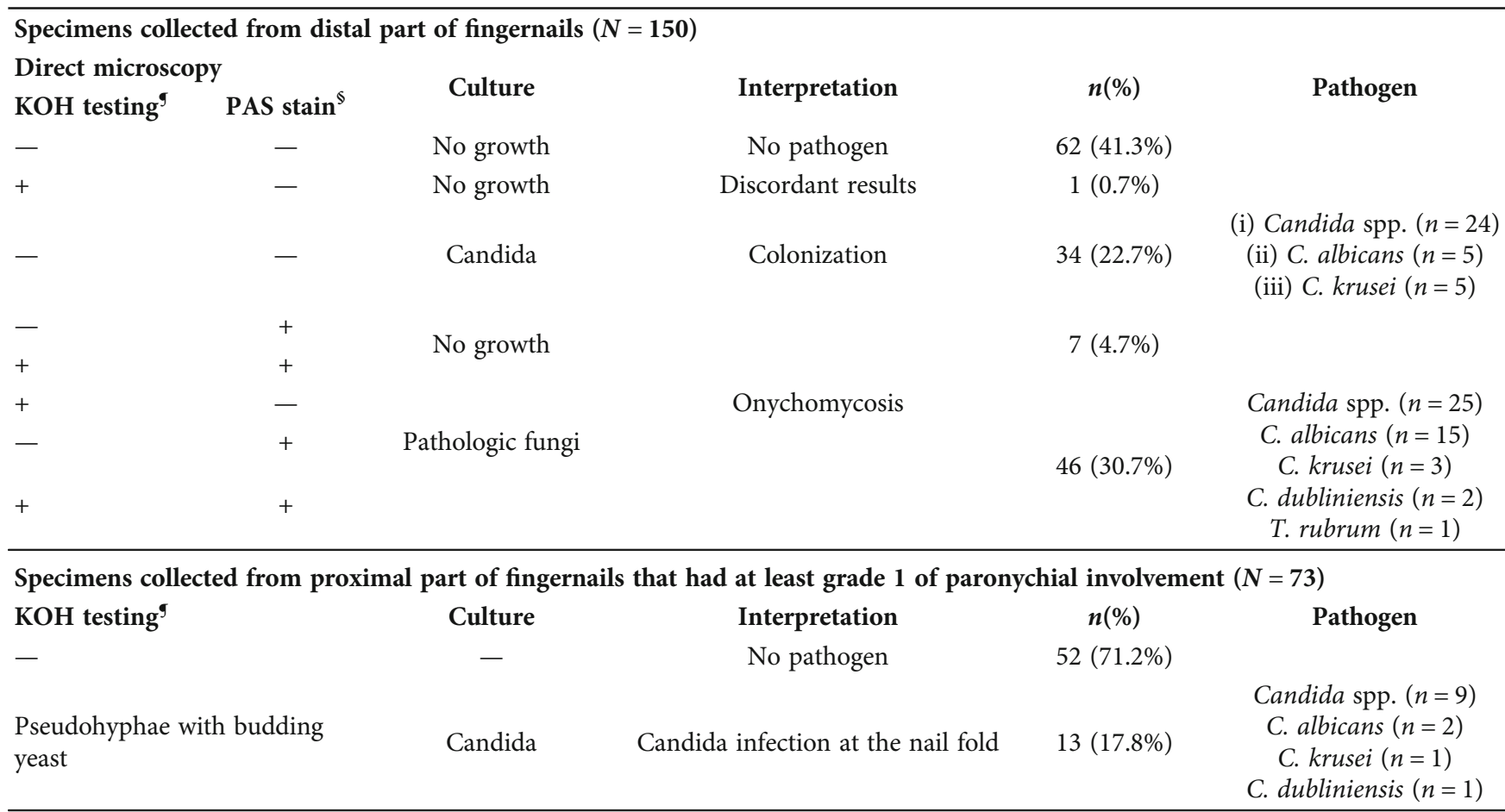

${ }^{9} \mathrm{KOH}:+$ indicates pseudohyphae with budding yeast or septate hyphae. ${ }^{{ }^{5} \mathrm{PAS}}$ + + indicates the presence of septate hyphae invading the nail plate or thicker tortuous wall hyphae or pseudohyphae with budding yeast.

this study provided written informed consent. The protocol for this study was approved by the Siriraj Institutional Review Board (SIRB) (COA no. Si.844/2018).

Demographic and clinical data, including gender, age, body mass index (BMI), duration of psoriasis, underlying diseases, psoriasis type, having or not having psoriatic arthritis, occupation, dominant hand side, and handwashing frequency, were recorded. Current psoriasis severity was assessed using the Psoriasis Area and Severity Index (PASI). The most severely affected fingernail of each hand was assessed using the Nail Psoriasis Severity Index (NAPSI) and the Nijmegen-Nail Psoriasis Activity Index tool (N-NAIL) [16, 17]. For NAPSI, the nail was divided into four quadrants, after which each quadrant was evaluated for the presence or absence of any of eight parameters indicating nail bed and/or nail matrix pathologies. The NAPSI score for one fingernail ranged from 0 to 8 [16]. For N-NAIL, the five evaluated parameters include onycholysis/oil drop, pitting, crumbling, Beau lines, and subungual hyperkeratosis. A score of 0 indicates no nail involvement, whereas a score of 3 represents maximum severity for each parameter for a total possible score of 15 for each fingernail [17]. The skin around each fingernail was evaluated for paronychia, and each nail was evaluated, as follows: grade 1 = redness and swelling of the nail folds; grade 2 = pronounced redness and swelling of the nail folds; grade 3 = redness and swelling of the nail folds with no cuticle; grade $4=$ redness and swelling of the nail folds, no cuticle, and tenderness and pain; and grade 5 = grade 4 plus acute paronychia on top of chronic paronychia [18].
Specimens obtained from the distal part of the most severely affected psoriatic nail from each hand were tested for onychomycosis using potassium hydroxide $(\mathrm{KOH})$, nail clipping for periodic acid-Schiff stain (PAS) technique, and fungal culture on Sabouraud dextrose agar and chloramphenicol media with and without cycloheximide (HiMedia Laboratories, Mumbai, India). Specimen collection and interpretation of $\mathrm{KOH}$ examination, PAS stain, and fungal cultures were performed by experienced technicians and one dermatopathologist. Cultures were incubated at $30^{\circ} \mathrm{C}$ and examined weekly up to 4 weeks. Chromogenic Candida Agar (Oxoid, Basingstoke, UK) was used to identify C. tropicalis, C. krusei, C. albicans, and C. dubliniensis on the basis of the morphology and color of the colonies [19]. Blue and brown/pink colonies indicate C. tropicalis and C. krusei, respectively. Green colonies indicate C. albicans or $C$ dubliniensis. The ability to grow at $42^{\circ} \mathrm{C}$ differentiates $C$. albicans (growth) from C. dubliniensis (no growth) [20]. Other Candida species that developed natural, mauve, or rose colors would be referred to Candida spp. in this study.

Specimens were collected from the nail bed as proximally to the cuticle as possible using a scalpel blade. Onychomycosis was diagnosed if (i) culture was positive for pathologic fungi whether direct microscopy $(\mathrm{KOH}$ and PAS) was positive or negative or if (ii) culture was negative, but PAS stain revealed pathological forms of fungal infection whether direct $\mathrm{KOH}$ examination was positive or negative. Fungal colonization of the nail was diagnosed if culture was positive for Candida species, but direct microscopy was negative. If the results were negative for all three 


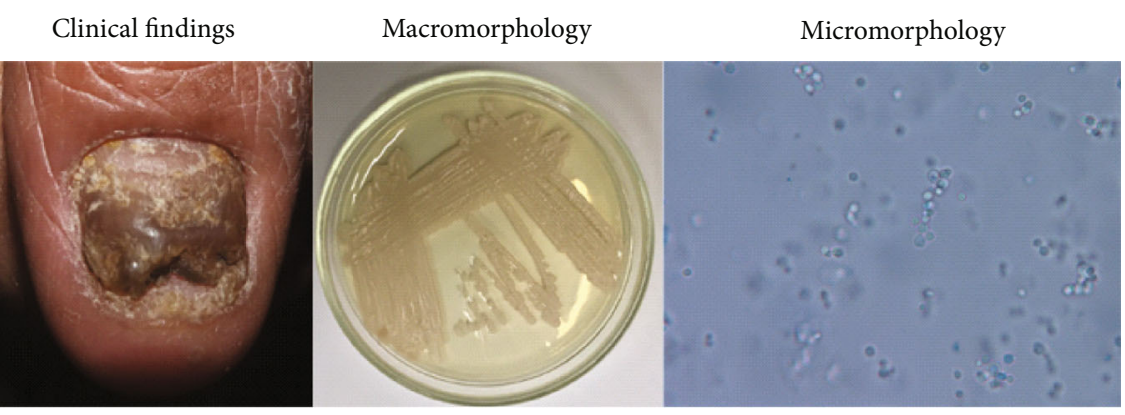

(a)
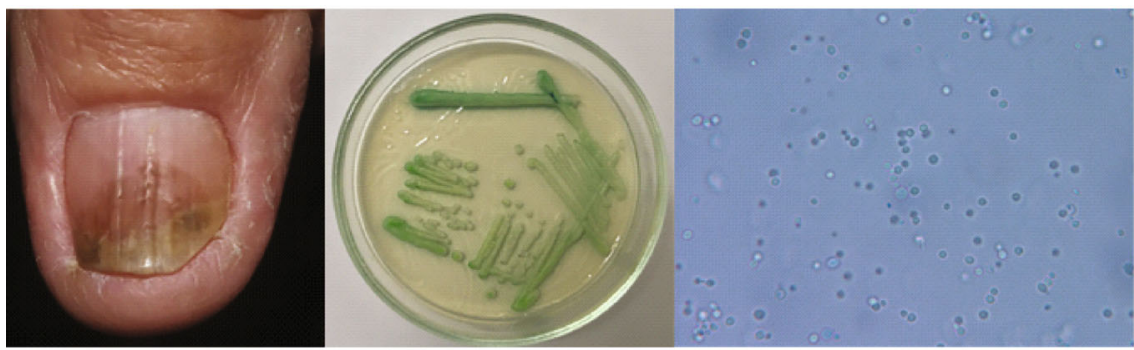

(b)

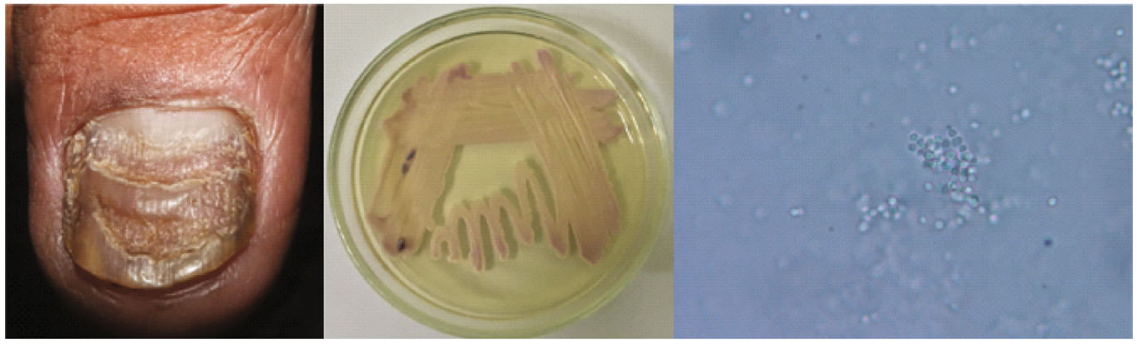

(c)
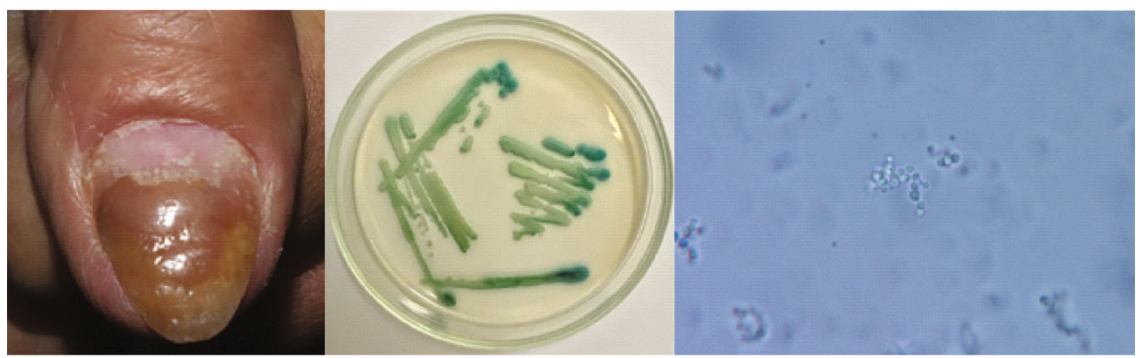

(d)

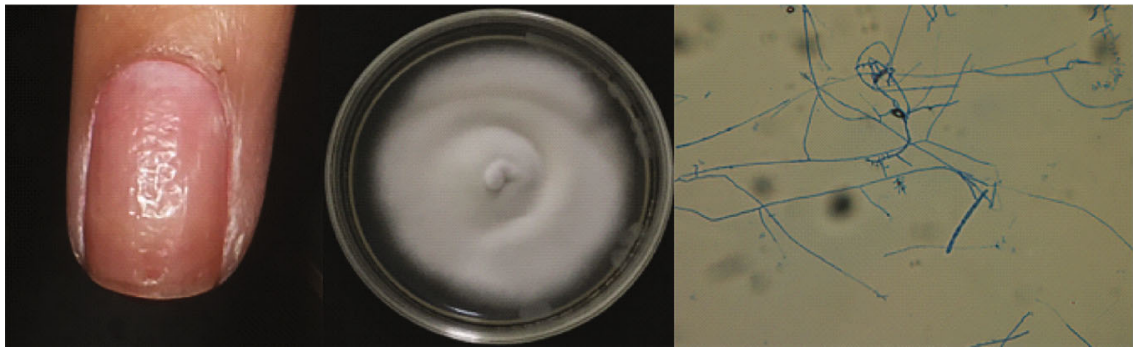

(e)

FIGURE 1: Representative pictures of clinical findings of psoriatic nail with fungal infection. Culture and microscopic identification from specimens collected from the distal part of psoriatic nail were also demonstrated. The fungi were inoculated onto the Chromogenic Candida Agar (a-d) and the Sabouraud dextrose agar with chloramphenicol (e) and inoculated at $30^{\circ} \mathrm{C}$ for 4 weeks. (a) Candida spp., (b) C. albicans, (c) C. krusei, (d) C. dubliniensis, and (e) Trichophyton rubrum. 
TABLE 3: Analysis for risk factors independently associated with onychomycosis in patients with nail psoriasis.

\begin{tabular}{|c|c|c|c|c|}
\hline & \multicolumn{2}{|l|}{ Univariate analysis } & \multicolumn{2}{|l|}{ Multivariate analysis } \\
\hline & $\begin{array}{c}\text { Crude odds ratio } \\
\text { (95\% confidence interval) }\end{array}$ & $p$ value & $\begin{array}{c}\text { Adjusted odds ratio } \\
\text { (95\% confidence interval) }\end{array}$ & $p$ value \\
\hline Male gender & $0.68(0.27-1.70)$ & 0.411 & & \\
\hline Concomitant diabetes mellitus & $6.06(1.53-24.02)$ & 0.010 & $7.04(1.44-34.50)$ & 0.016 \\
\hline Duration of psoriasis $\geq 10$ years & $0.99(0.37-2.70)$ & 0.989 & & \\
\hline Handwashing frequency $\geq 6$ times/day & $1.83(0.73-4.58)$ & 0.200 & & \\
\hline Occupation that increased the risk of hand exposure to water & $5.05(1.89-13.52)$ & 0.001 & $4.12(1.36-12.51)$ & 0.012 \\
\hline Nail Psoriasis Severity Index score $\geq 4$ & $1.30(0.52-3.24)$ & 0.570 & & \\
\hline Nijmegen - Nail Psoriasis Activity Index score $\geq 4$ & $1.70(0.65-4.46)$ & 0.281 & & \\
\hline Topical treatment: methotrexate & $3.19(1.00-10.17)$ & 0.050 & $2.12(0.55-8.16)$ & 0.275 \\
\hline Biologic $^{\#}$ & $0.58(0.18-1.91)$ & 0.372 & $0.46(0.12-1.86)$ & 0.277 \\
\hline Biologic treatment ${ }^{\#}$ : topical & $1.71(0.53-5.60)$ & 0.372 & $2.16(0.54-8.65)$ & 0.277 \\
\hline Methotrexate & $5.46(1.63-18.36)$ & 0.006 & $4.57(1.11-18.93)$ & 0.036 \\
\hline Pitted nails & $1.51(0.54-4.23)$ & 0.434 & & \\
\hline Leukonychia & $1.14(0.46-2.83)$ & 0.785 & & \\
\hline Crumbling & $1.69(0.65-4.41)$ & 0.282 & & \\
\hline Onycholysis & $0.46(0.10-2.07)$ & 0.311 & & \\
\hline Subungual hyperkeratosis & $1.09(0.43-2.77)$ & 0.850 & & \\
\hline Paronychial involvement & $0.90(0.36-2.25)$ & 0.817 & & \\
\hline Candida infection in the oral cavity & $3.33(0.92-12.01)$ & 0.066 & $2.82(0.59-13.39)$ & 0.192 \\
\hline
\end{tabular}

Variables with a $p$ value $<0.20$ in univariate analysis were included in multivariate analysis. A $p$ value $<0.05$ in multivariate analysis was considered statistically significant. "Interleukin- (IL-) 17 inhibitors, antitumor necrosis factor, and anti-IL 12/23 were used in 20, 4, and 1 patient, respectively.

techniques (KOH, PAS, culture), negative pathogen would be diagnosed [21]. Patients with a diagnosis of onychomycosis were defined as patients who had onychomycosis of the right and/or left hand. $\mathrm{KOH}$ examination and culture were also performed at the proximal nail fold. Candida infection of the nail fold was diagnosed if there was at least grade one of paronychial involvement and positive result for both direct microscopy $(\mathrm{KOH})$ and culture. Candida infection in the oral cavity was also recorded. Oral candidiasis was diagnosed if both $\mathrm{KOH}$ and culture showed a positive result for Candida infection.

2.1. Sample Size Calculation and Statistical Analysis. The sample size for each group was calculated using $\lambda^{2}$. Previous studies reported the prevalence of onychomycosis in psoriasis patients treated with topical medication, methotrexate, and biologic agents to be $20 \%, 50 \%$, and $13 \%$, respectively [14, 15, 21]. Using a 2-sided type I error of 0.05 and $80 \%$ power, a sample of 25 patients per group was required (75 patients in total).

Descriptive statistics were used to summarize patient demographic and clinical characteristics. Data are described as mean plus/minus standard deviation (SD) for continuous data with normal distribution and as median and interquartile range (IQR) for nonnormally distributed continuous data. Categorical data are described as number and percentage. The results of univariate analysis and multivariate analysis are shown as odds ratio and adjusted odds ratio with their 95\% confidence intervals, respectively. Statistical Package for the Social Sciences 18.0 (SPSS, Inc., Chicago, IL, USA) was used for data analysis, and a $p$ value less than 0.05 was considered to be statistically significant.

\section{Results}

Among the 75 enrolled patients, the gender distribution was almost equal, and the mean age was $46.8 \pm 15.3$ years. The most common underlying disease was dyslipidemia (24.0\%), followed by hypertension $(22.7 \%)$, diabetes mellitus (18.7\%), metabolic syndrome (13.3\%), and obesity (12.0\%). Table 1 shows the demographic and clinical characteristics of the included patients. Most patients had psoriasis without psoriatic arthritis (82.7\%). Forty patients (53.3\%) had an occupation that did not expose the patient to an increased risk of hand exposure to water. The median handwashing frequency of patients was 6 times/day, with a minimum of 2 times/day and a maximum of 20 times/day. Patterns of nail involvement and nail pathology were similar between the right hand and the left hand. Thirteen patients $(17.3 \%)$ had oral candidiasis. We also found significant correlation between oral candidiasis and Candida infection of the nails ( $p=0.015$, data not shown).

Of 150 fingernails ( 1 from each hand of each of 75 patients), the thumb was the most severely affected fingernail on both hands. Pitted nail and onycholysis were the most common abnormalities of the nail matrix and nail bed, respectively. Seventy-three fingernails had paronychial involvement with $71.2 \%, 26.0 \%$, and $2.7 \%$ of grades 1,2 , and 3 paronychial involvement, respectively. Fifty-three $(35.3 \%)$ fingernails and $34(22.7 \%)$ fingernails had 
TABLE 4: Characteristics of nail psoriasis patients compared among psoriasis treatment regimens.

\begin{tabular}{|c|c|c|c|c|}
\hline \multirow[b]{2}{*}{ Characteristics } & \multicolumn{3}{|c|}{ Number of patients $(N=75)$} & \multirow[b]{2}{*}{ pvalue } \\
\hline & $\begin{array}{l}\text { Topical treatment } \\
\qquad(n=25)\end{array}$ & $\begin{array}{l}\text { Methotrexate } \\
\quad(n=25)\end{array}$ & $\begin{array}{l}\text { Biologic agents } \\
\quad(n=25)\end{array}$ & \\
\hline \multicolumn{5}{|l|}{ Gender, $n(\%)$} \\
\hline Female & $9(36.0 \%)$ & $13(52.0 \%)$ & $16(64.0 \%)$ & \\
\hline Male & $16(64.0 \%)$ & $12(48.0 \%)$ & $9(36.0 \%)$ & \\
\hline Age (years), mean $\pm \mathrm{SD}$ & $48.1 \pm 17.2$ & $47.8 \pm 11.3$ & $44.4 \pm 16.8$ & 0.403 \\
\hline Body mass index $\left(\mathrm{kg} / \mathrm{m}^{2}\right)$, mean $\pm \mathrm{SD}$ & $23.6 \pm 4.0$ & $25.6 \pm 5.4$ & $25.9 \pm 5.2$ & 0.042 \\
\hline Concomitant diabetes mellitus, $n(\%)$ & $2(8.0 \%)$ & $7(28.0 \%)$ & $5(20.0 \%)$ & 0.226 \\
\hline \multicolumn{5}{|l|}{ Occupation, $n(\%)$} \\
\hline Increased risk of hand exposure to water & $9(36.0 \%)$ & $17(68.0 \%)$ & $9(36.0 \%)$ & 0.032 \\
\hline \multicolumn{5}{|l|}{ Nail severity, median (IQR) } \\
\hline Nail Psoriasis Severity Index score & $3.0(2.0,4.0)$ & $3.0(2.0,4.0)$ & $4.0(2.0,4.0)$ & 0.979 \\
\hline Nijmegen-Nail Psoriasis Activity Index score & $4.0(3.0,5.0)$ & $4.0(3.0,5.0)$ & $4.0(3.0,5.0)$ & 0.333 \\
\hline \multicolumn{5}{|l|}{ Patients with diagnosis of onychomycosis } \\
\hline No & $15(60.0 \%)$ & $8(32.0 \%)$ & $18(72.0 \%)$ & 0.014 \\
\hline \multirow[t]{2}{*}{ Yes (right or left hand) } & $10(40.0 \%)$ & $17(68.0 \%)$ & $7(28.0 \%)$ & \\
\hline & \multicolumn{4}{|c|}{ Number of fingernails $(N=150)$} \\
\hline Characteristics & $\begin{array}{l}\text { Topical treatment } \\
\qquad(n=50)\end{array}$ & $\begin{array}{l}\text { Methotrexate } \\
\quad(n=50)\end{array}$ & $\begin{array}{l}\text { Biologic agents }^{\#} \\
\quad(n=50)\end{array}$ & pvalue \\
\hline Candida infection of the nails & $16(32.0 \%)$ & $25(50.0 \%)$ & $9(18.0 \%)$ & 0.030 \\
\hline Candida colonization of the nails & $11(22.0 \%)$ & $8(16.0 \%)$ & $15(30.0 \%)$ & 0.245 \\
\hline Candida infection of the nail folds & $7(14.0 \%)$ & $8(16.0 \%)$ & $6(12.0 \%)$ & 0.847 \\
\hline Candida infection of the nails and nail folds & $7(14.0 \%)$ & $7(14.0 \%)$ & $6(12.0 \%)$ & 1.000 \\
\hline
\end{tabular}

${ }^{\#}$ Interleukin- (IL-) 17 inhibitors, antitumor necrosis factor, and anti-IL 12/23 were used in 20, 4, and 1 patient, respectively. A $p$ value $<0.05$ indicates statistical significance. Abbreviations: SD: standard deviation; IQR: interquartile range.

onychomycosis and Candida colonization, respectively (Table 2). The most common organism causing onychomycosis was Candida spp., followed by Trichophyton rubrum. Candida infection of the nail fold was found in $13(17.8 \%)$ of the 73 fingernails with paronychial involvement. The photos of clinical findings of nail psoriasis with fungal infection and macro and micromorphology of the fungal isolates are shown as Figure 1.

Univariate analysis showed concomitant diabetes, occupation that might increase the risk of hand exposure to water, and methotrexate to be significant risk factors associated with onychomycosis (Table 3), and all three of those factors remained statistically significant in multivariate analysis. When we excluded patients with diabetes and reanalyzed our data, wet-work exposure and methotrexate were significant risk factors for the development of onychomycosis in univariate analysis $(p=0.002, p=0.028)$, and wet-work exposure remained as the only risk factor independently associated with onychomycosis in multivariate analysis $(p=0.007)$. Table 4 shows that the prevalence of onychomycosis was highest in the methotrexate group (68\% in methotrexate treatment, $40 \%$ in topical treatment, and $28 \%$ in biologic treatment). However, the number of patients with diabetes and wet-work exposure was higher in the methotrexate group than in the other two groups. The risk of Candida colonization of the nail did not increase significantly in the biologic treatment group even though the main biologics used were interleukin-17 inhibitors.

\section{Discussion}

A systematic review in 2014 showed an increased prevalence of onychomycosis in psoriatic patients (18\%) compared to the prevalence in the general population (9.1\%); however, the high heterogeneity among the 10 included studies limits the reliability of their findings [22]. We reviewed the literature in the PubMed database using the keywords "prevalence," "onychomycosis," "psoriasis," and "treatment." Thirty studies were included and summarized, as shown in Table $5[2,6,13-15,21,23-46]$. It was shown that the prevalence of onychomycosis in psoriatic nails in Asian countries ranged from $20.3 \%$ (Kuwait) to $47.9 \%$ (India) compared to the prevalence of $18.0 \%$ (Belgium, Poland) to $62.0 \%$ (Bulgaria) in European countries [2, 6, 13-15, 23, $25,27-33,35,37,40,41,44-46]$. The prevalence of onychomycosis in controls with clinical abnormality in Asian countries ranged from 4\% (Pakistan) to $40.6 \%$ (Turkey) compared to the prevalence of $22.4 \%$ (Poland) to $51.3 \%$ (Italy) in European countries [2, 14, 15, 32, 33, 35, 37, 44-46]. The first study of the prevalence of onychomycosis in psoriasis patients at our center was published in 2018, and that study found a prevalence of $32.3 \%$ [23]. The 


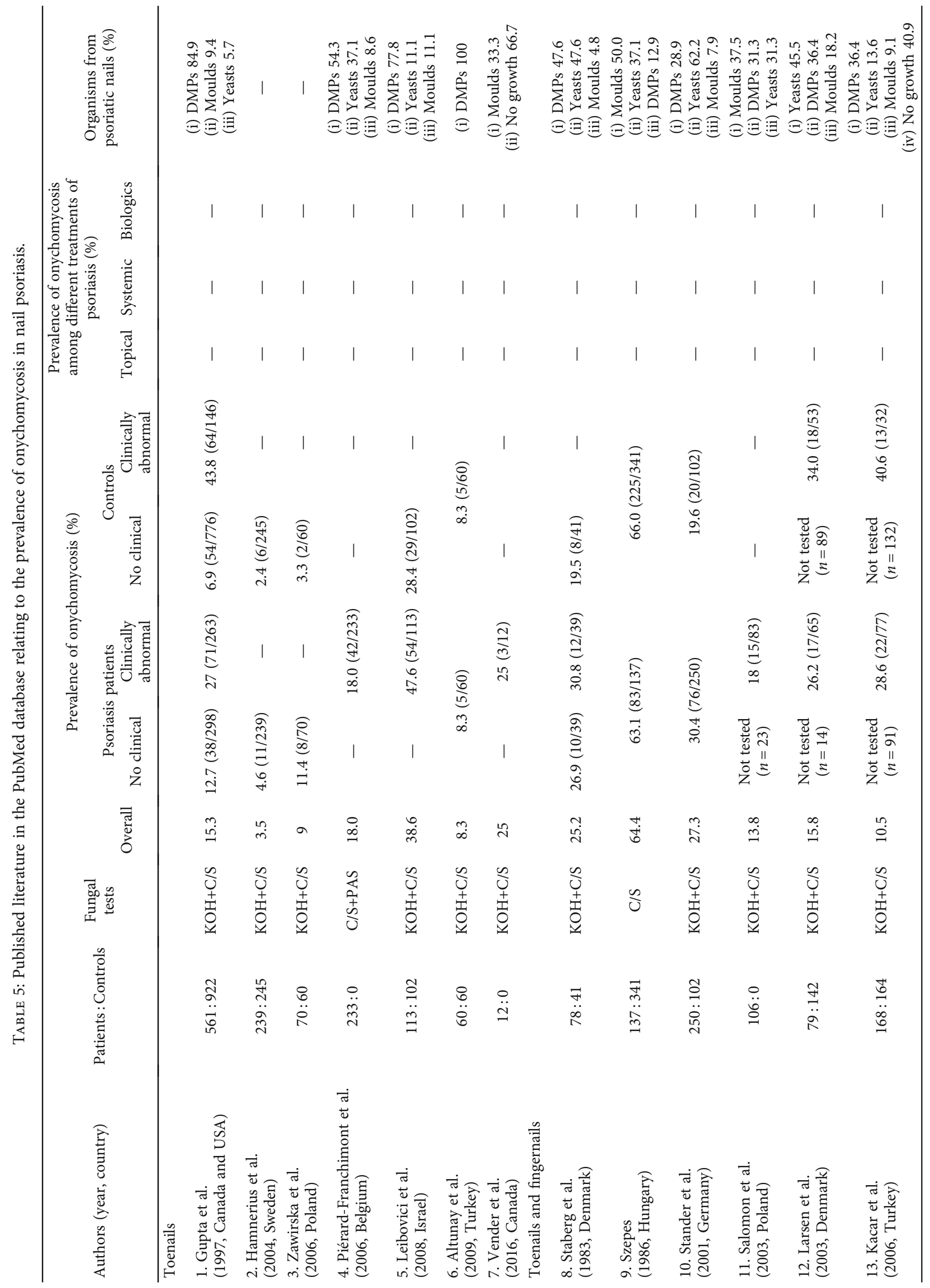




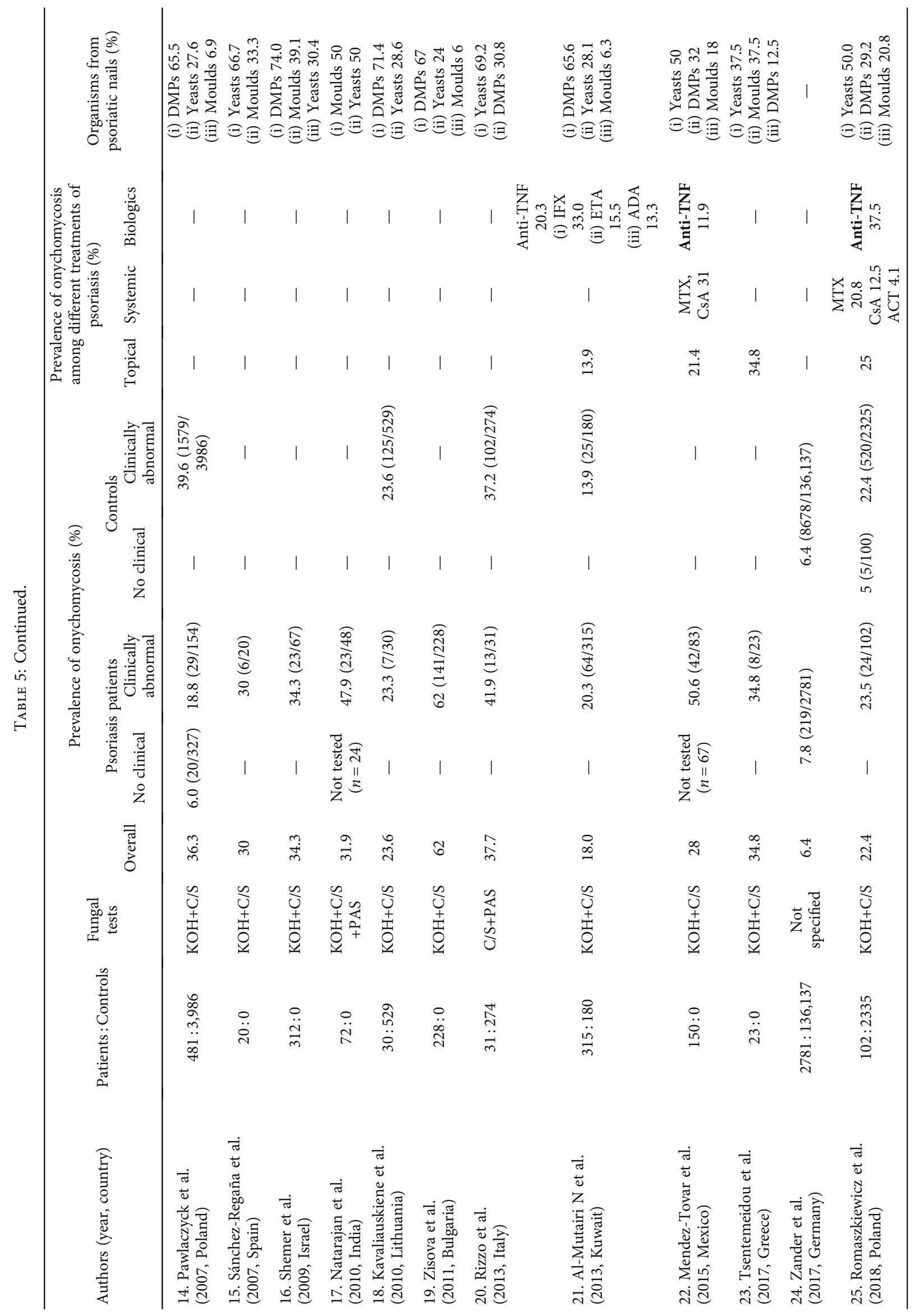




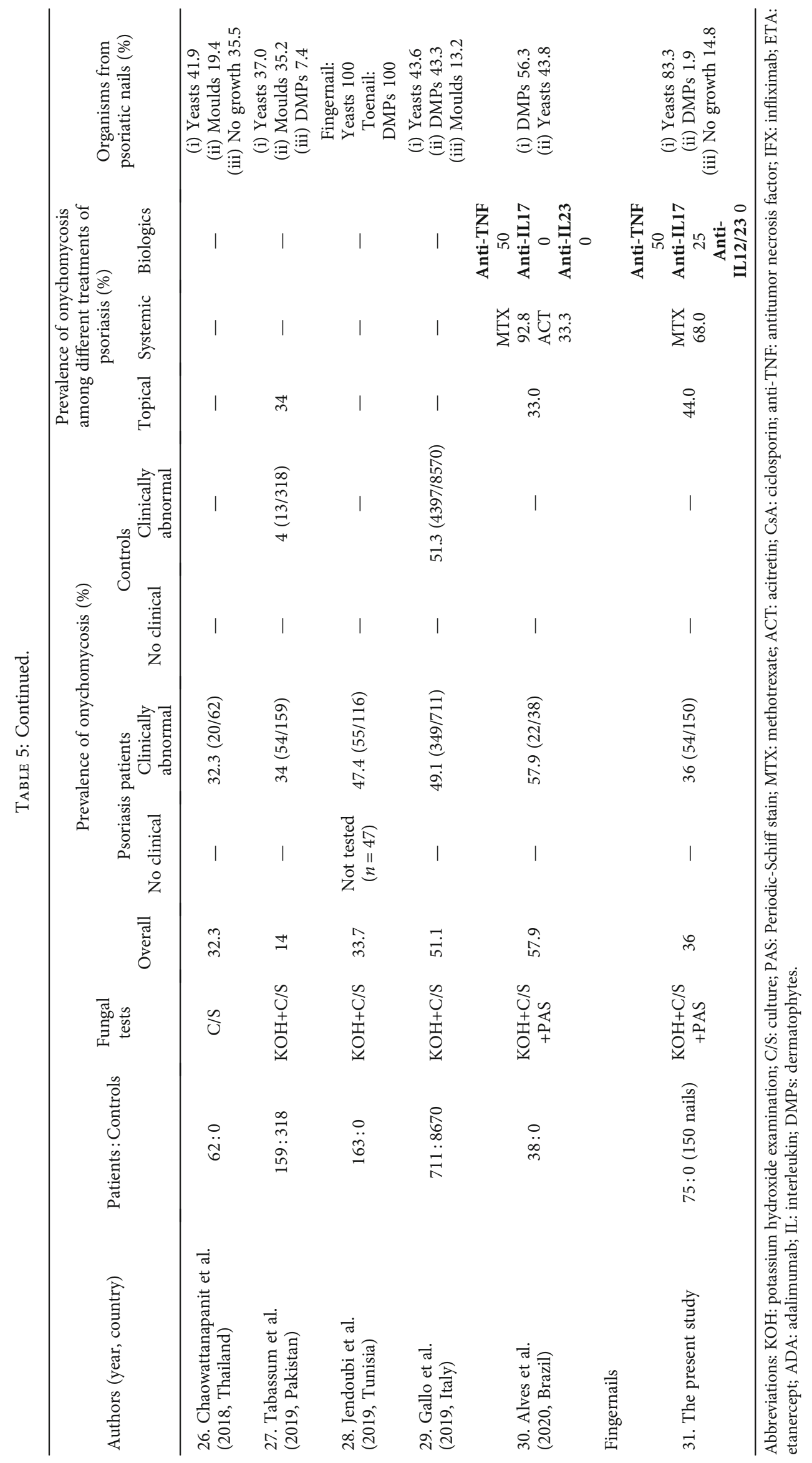


prevalence in this study was $35.3 \%$ (53/150), which is close to, but higher than that from the previous study. It seemed that the prevalence of onychomycosis in psoriatic patients in the present study was in a range of the reported prevalence of onychomycosis in controls with clinical nail abnormality in Asian countries [15, 35, 45].

Yeasts (Candida spp.) were more commonly identified in the present study, which is similar to several previous studies $[2,1314,23,24]$. Chadeganipour et al. used molecular technique to identify Candida species in clinical samples of patients with psoriasis [47]. Molecular technique can provide $95 \%$ sensitivity and $100 \%$ specificity [48]. In that study, C. parapsilosis was the most prevalent species among Candida species of fingernail infection and none of Candida species were albicans. A lower number of clinical samples and different technique to identify Candida species in Chadeganipour's study may explain the results different from our study [47].

Our study showed diabetes, methotrexate treatment, and wet-work exposure to be significant risk factors associated with onychomycosis. Methotrexate was at greater risk of developing onychomycosis compared to biologic treatment. The pathogenesis of nail psoriasis may increase or decrease the risk of onychomycosis. Generally, rapid nail growth, increase in antimicrobial peptides, and compact orthokeratotic nail plate will decrease the risk of onychomycosis in nail psoriasis [5]. However, other factors that increase the risk of onychomycosis also play an important role. Methotrexate can increase the risk of onychomycosis by immunosuppressive effect and slow the rate of nail growth [6].

Studies reporting the prevalence of onychomycosis in psoriasis patients who were on biologic treatment mainly included patients receiving anti-TNF treatment $[14,15,21$, 24]. Three out of four studies, including a randomized prospective study, showed a higher prevalence of onychomycosis in psoriatic patients treated with anti-TNF than in patients that received other types of treatments $[14,15,21$, 24]. There are some possible explanations why biologics did not increase the risk of onychomycosis in our study. First, the faster nail growth rate in psoriasis [49-51] acts as a protective factor against onychomycosis. Second, biologic treatment is more effective for treating nail psoriasis than methotrexate [52] because it reduces nail pathology and the opportunity of the fungi to invade the nail keratin. Third and last, the biologics most often used in our study were interleukin-17 inhibitors, which may have less immunosuppressive effect than anti-TNF.

4.1. Limitations. This study has some mentionable limitations. Even though we enrolled a sufficient number of patients to satisfy the minimum described by our sample size calculation, 75 patients represent a relatively small number of patients. Besides, only 20 patients were treated with interleukin-17 inhibitors, which could limit the generalizability of our results. Secondly, toenails were not included in this study since several other factors influence the risk of developing onychomycosis of the toenails compared to the fingernails. Thirdly, in cases of diagnostic discordance among the 3 diagnostic methods, a retest was not performed.
Our rationale for this is that all three diagnostic methods were performed by experienced technicians and one expert dermatopathologist. Finally, molecular biology testing, which provides high sensitivity, high specificity, and accurate identification of fungal species, could not be performed in this study due to its relatively high cost. Alternatively, our laboratory used a culture-based method to identify fungal species. Only C. albicans, C. dubliniensis, C. tropicalis, and C. krusei could be identified. This is why we reported the other groups of Candida as Candida spp.

\section{Conclusions}

The prevalence of onychomycosis in nail psoriasis in this study was $35.3 \%$. Among the three different psoriasis treatment groups, the prevalence of onychomycosis was significantly higher in the methotrexate treatment group than in the topical treatment and biologic treatment groups. Several factors can affect the risk of onychomycosis, including occupation, psoriasis treatment, host status, and nail growth rate. Molecular identification would be the best method to elucidate the etiology and establish an epidemiological inference with previous findings in the literature.

\section{Data Availability}

The data used to support the findings of this study are included within the article.

\section{Conflicts of Interest}

All authors declare no conflicts of interest.

\section{Acknowledgments}

The authors gratefully acknowledge the patients that participated in this study, Ms. Lalita Matthapan, Mr. Waranyoo Prasong, and Ms. Kamonpan Lertrujiwanit of the Department of Dermatology Faculty of Medicine Siriraj Hospital, Mahidol University, for conducting mycological examinations, and Asst. Prof. Dr. Chulaluk Komoltri for statistical advice. This study was supported by grants from the Dermatological Society of Thailand.

\section{References}

[1] V. Caputo, C. Strafella, A. Termine et al., "Overview of the molecular determinants contributing to the expression of psoriasis and psoriatic arthritis phenotypes," Journal of Cellular and Molecular Medicine, vol. 24, no. 23, pp. 13554-13563, 2020.

[2] L. Gallo, E. Cinelli, G. Fabbrocini, and M. Vastarella, "A 15year retrospective study on the prevalence of onychomycosis in psoriatic vs non-psoriatic patients: a new European shift from dermatophytes towards yeast," Mycoses, vol. 62, no. 8, pp. 659-664, 2019.

[3] A. Ventura, M. Mazzeo, R. Gaziano, M. Galluzzo, L. Bianchi, and E. Campione, "New insight into the pathogenesis of nail psoriasis and overview of treatment strategies," Drug Design, Development and Therapy, vol. Volume 11, pp. 2527-2535, 2017. 
[4] I. Raposo and T. Torres, "Psoriasis ungueal como factor predictivo del desarrollo de artritis prosiasica," Actas Dermo-Sifiliográficas, vol. 106, no. 6, pp. 452-457, 2015.

[5] D. Rigopoulos, V. Papanagiotou, R. Daniel 3rd, and B. M. Piraccini, "Onychomycosis in patients with nail psoriasis: a point to point discussion," Mycoses, vol. 60, no. 1, pp. 6-10, 2017.

[6] L. Zisova, V. Valtchev, E. Sotiriou, D. Gospodinov, and G. Mateev, "Onychomycosis in patients with psoriasis-a multicentre study," Mycoses, vol. 55, no. 2, pp. 143-147, 2012.

[7] E. Campione, E. J. Paterno, L. Diluvio et al., "Tazarotene as alternative topical treatment for onychomycosis," Drug Design, Development and Therapy, vol. 9, pp. 879-886, 2015.

[8] L. Diluvio, E. Campione, E. J. Paternò, C. Mordenti, M. El Hachem, and S. Chimenti, "Childhood nail psoriasis: a useful treatment with tazarotene $0.05 \%$," Pediatric Dermatology, vol. 24, no. 3, pp. 332-333, 2007.

[9] T. Cosio, M. Di Prete, R. Gaziano et al., "Trifarotene: a current review and perspectives in dermatology," Biomedicine, vol. 9, p. 237, 2021.

[10] E. Campione, T. Cosio, C. Lanna et al., "Predictive role of vitamin A serum concentration in psoriatic patients treated with IL-17 inhibitors to prevent skin and systemic fungal infections," Journal of Pharmacological Sciences, vol. 144, no. 1, pp. 52-56, 2020.

[11] E. Campione, R. Gaziano, D. Marino, and A. Orlandi, "Fungistatic activity of all-trans retinoic acid against Aspergillus fumigatus and Candida albicans," Drug Design, Development and Therapy, vol. 10, pp. 1551-1555, 2016.

[12] C. Lanna, G. M. Cesaroni, S. Mazzilli et al., "Nails as immuneprivileged sites: a case of disabling acrodermatitis continua of Hallopeau successfully treated with apremilast," Dermatologic Therapy, vol. 32, article e12946, 2019.

[13] A. Tsentemeidou, T. A. Vyzantiadis, A. Kyriakou, D. Sotiriadis, and A. Patsatsi, "Prevalence of onychomycosis among patients with nail psoriasis who are not receiving immunosuppressive agents: results of a pilot study," Mycoses, vol. 60, no. 12, pp. 830-835, 2017.

[14] A. Romaszkiewicz, B. Bykowska, M. Zabłotna, M. Sobjanek, M. Sławińska, and R. J. Nowicki, “The prevalence and etiological factors of onychomycosis in psoriatic patients," Postepy Dermatol Alergol., vol. 35, no. 3, pp. 309-313, 2018.

[15] N. Al-Mutairi, T. Nour, and D. Al-Rqobah, "Onychomycosis in patients of nail psoriasis on biologic therapy: a randomized, prospective open label study comparing etanercept, infliximab and adalimumab," Expert Opinion on Biological Therapy, vol. 13, no. 5, pp. 625-629, 2013.

[16] P. Rich and R. K. Scher, "Nail psoriasis severity index: a useful tool for evaluation of nail psoriasis," Journal of the American Academy of Dermatology, vol. 49, no. 2, pp. 206-212, 2003.

[17] K. M. Klaassen, P. C. van de Kerkhof, M. T. Bastiaens, L. G. Plusjé, R. L. Baran, and M. C. Pasch, "Scoring nail psoriasis," Journal of the American Academy of Dermatology, vol. 70, no. 6, pp. 1061-1066, 2014.

[18] C. R. Daniel 3rd, M. Iorizzo, B. M. Piraccini, and A. Tosti, "Grading simple chronic paronychia and onycholysis," International Journal of Dermatology, vol. 45, no. 12, pp. 14471448, 2006.

[19] Oxoid product list, 2012, Available from: http://www.oxoid .com. doi:10.1094/PDIS-11-11-0999-PDN. Accessed October 2021. [CrossRef].

[20] K. Tintelnot, G. Haase, M. Seibold et al., "Evaluation of phenotypic markers for selection and identification of Candida dubliniensis," Journal of Clinical Microbiology, vol. 38, no. 4, pp. 1599-1608, 2000.

[21] N. C. P. O. Praeiro Alves, T. D. Moreira, L. D. Malvino et al., "Onychomycosis in psoriatic patients with nail disorders: aetiological agents and immunosuppressive therapy," Dermatology Research and Practice, vol. 2020, 7 pages, 2020.

[22] K. M. Klaassen, M. G. Dulak, P. C. van de Kerkhof, and M. C. Pasch, "The prevalence of onychomycosis in psoriatic patients: a systematic review," Journal of the European Academy of Dermatology and Venereology, vol. 28, no. 5, pp. 533-541, 2014.

[23] S. Chaowattanapanit, P. Pattanaprichakul, C. Leeyaphan et al., "Coexistence of fungal infections in psoriatic nails and their correlation with severity of nail psoriasis," Indian Dermatology Online Journal, vol. 9, no. 5, pp. 314-317, 2018.

[24] L. J. Méndez-Tovar, A. Arévalo-López, S. Domínguez-Aguilar et al., "Onychomycosis frequency in psoriatic patients in a tertiary care hospital," Revista Médica del Instituto Mexicano del Seguro Social, vol. 53, no. 3, pp. 374-379, 2015.

[25] C. Piérard-Franchimont, J. E. Arrese, T. Hermanns-Lê, and G. E. Piérard, "Épidemiologie des onychomycoses evaluees par histomycologie chez des patients psoriasiques," Journal de Mycologie Médicale., vol. 16, no. 3, pp. 159-162, 2006.

[26] Z. T. Altunay, M. Ilkit, and Y. Denli, "Investigation of tinea pedis and toenail onychomycosis prevalence in patients with psoriasis," Mikrobiyoloji Bülteni, vol. 43, no. 3, pp. 439-447, 2009.

[27] R. Vender and R. Vender, "Psoronychomycosis: a new term for an old problem," Journal of Cutaneous Medicine and Surgery, vol. 20, no. 3, pp. 279-280, 2016.

[28] J. Salomon, J. C. Szepietowski, and A. Proniewicz, "Psoriatic nails: a prospective clinical study," Journal of Cutaneous Medicine and Surgery, vol. 7, no. 4, pp. 317-321, 2003.

[29] M. L. Sánchez-Regaña, S. Videla, J. Villoria et al., "Prevalence of fungal involvement in a series of patients with nail psoriasis," Clinical and Experimental Dermatology, vol. 33, no. 2, pp. 194-195, 2008.

[30] A. Shemer, H. Trau, B. Davidovici, M. H. Grunwald, and B. Amichai, "Onychomycosis in psoriatic patients - rationalization of systemic treatment," Mycoses, vol. 53, no. 4, pp. 340-343, 2010.

[31] D. M. Thappa, R. Singh, S. K. Verma, V. Natarajan, and A. K. Nath, "Coexistence of onychomycosis in psoriatic nails: a descriptive study," Indian Journal of Dermatology, Venereology and Leprology, vol. 76, no. 6, p. 723, 2010.

[32] S. Kavaliauskiene, R. Povilionyte, J. Jakubovskiene et al., "Relationships between the incidence of onychomycosis and nail psoriasis," Medicina (Kaunas, Lithuania), vol. 46, pp. 180$184,2010$.

[33] D. Rizzo, R. Alaimo, G. Tilotta, F. Dinotta, and M. R. Bongiorno, "Incidence of onychomycosis among psoriatic patients with nail involvement: a descriptive study," Mycoses, vol. 56, no. 4, pp. 498-499, 2013.

[34] N. Zander, I. Schäfer, M. Radtke, A. Jacobi, H. Heigel, and M. Augustin, "Dermatological comorbidity in psoriasis: results from a large-scale cohort of employees," Archives of Dermatological Research, vol. 309, no. 5, pp. 349-356, 2017.

[35] S. Tabassum, A. Rahman, S. Awan et al., "Factors associated with onychomycosis in nail psoriasis: a multicenter study in Pakistan," International Journal of Dermatology, vol. 58, no. 6, pp. 672-678, 2019.

[36] F. Jendoubi, I. Ben Lagha, F. Rabhi et al., "Nail involvement in psoriatic patients and association with onychomycosis: results 
from a cross-sectional study performed in a military hospital in Tunisia," Skin Appendage Disord., vol. 5, no. 5, pp. 299303, 2019.

[37] A. K. GUPTA, C. W. LYNDE, H. C. JAIN et al., "A higher prevalence of onychomycosis in psoriatics compared with non-psoriatics: a multicentre study," The British Journal of Dermatology, vol. 136, no. 5, pp. 786-789, 1997.

[38] N. Hamnerius, J. Berglund, and J. Faergemann, "Pedal dermatophyte infection in psoriasis," The British Journal of Dermatology, vol. 150, no. 6, pp. 1125-1128, 2004.

[39] A. Zawirska, Z. Adamski, and L. Kurek, "Dermatophyte infections in psoriatic patients," Mikologia Lekarska, vol. 13, pp. 287-290, 2006.

[40] V. Leibovici, K. Hershko, A. Ingber, M. Westerman, N. Leviatan-Strauss, and M. Hochberg, "Increased prevalence of onychomycosis among psoriatic patients in Israel," Acta Dermato-Venereologica, vol. 88, no. 1, pp. 31-33, 2008.

[41] B. Staberg, M. Gammeltoft, and P. Onsberg, "Onychomycosis in patients with psoriasis," Acta Dermato-Venereologica, vol. 63 , no. 5, pp. 436-438, 1983.

[42] E. Szepes, "Mycotic infections of psoriatic nails," Mykosen, vol. 29, no. 2, pp. 82-84, 1986.

[43] H. Ständer, M. Ständer, and S. Nolting, "Incidence of fungal involvement in nail psoriasis," Der Hautarzt, vol. 52, no. 5, pp. 418-422, 2001.

[44] G. K. Larsen, M. Haedersdal, and E. L. Svejgaard, "The prevalence of onychomycosis in patients with psoriasis and other skin diseases," Acta Dermato-Venereologica, vol. 83, no. 3, pp. 206-209, 2003.

[45] N. Kaçar, S. Ergin, C. Ergin, B. S. Erdogan, and I. Kaleli, "The prevalence, aetiological agents and therapy of onychomycosis in patients with psoriasis: a prospective controlled trial," Clinical and Experimental Dermatology, vol. 32, no. 1, pp. 1-5, 2007.

[46] M. Pawlaczyk, A. Rokowska, I. Chmielewska, D. Janicka, and A. Gutowska-Ryters, "Does onychomycosis more frequently affect patients suffering from psoriasis?," Mikol Lek., vol. 13, p. 82, 2006.

[47] M. Chadeganipour, S. Shadzi, and R. Mohammadi, "Fungal infections among psoriatic patients: etiologic agents, comorbidities, and vulnerable population," Autoimmune Diseases., vol. 2021, pp. 1-7, 2021.

[48] M. Ghannoum, P. Mukherjee, N. Isham, B. Markinson, J. D. Rosso, and L. Leal, "Examining the importance of laboratory and diagnostic testing when treating and diagnosing onychomycosis," International Journal of Dermatology, vol. 57, no. 2, pp. 131-138, 2018.

[49] M. Paduszynska, W. Baranska-Rybak, and S. Bielińska, “The influence of antimicrobial peptides on Candida biofilm," Alergia Astma Immunologia., vol. 15, pp. 220-225, 2010.

[50] M. Marcinkiewicz and S. Majewski, "The role of antimicrobial peptides in chronic inflammatory skin diseases," Postepy Dermatol Alergol, vol. 1, pp. 6-12, 2016.

[51] I. Błażewicz, M. Jaśkiewicz, L. Piechowicz, W. Kamysz, R. Nowicki, and W. Barańska-Rybak, "The role of antimicrobial peptides in selected dermatoses," Przeglad Dermatologiczny, vol. 3, pp. 227-232, 2016.

[52] B. E. Elewski, C. S. Baker, J. J. Crowley et al., "Adalimumab for nail psoriasis: efficacy and safety over 52 weeks from a phase-3, randomized, placebo-controlled trial," Journal of the European Academy of Dermatology and Venereology, vol. 33, no. 11, pp. 2168-2178, 2019. 